\title{
Multimodal nocturnal seizure detection in a residential care setting
}

\section{A long-term prospective trial}

Johan Arends, MD, PhD, Roland D. Thijs, MD, PhD, Thea Gutter, MSc, Constantin Ungureanu, MSc, PhD, Pierre Cluitmans, MSc, PhD, Johannes Van Dijk, PhD, Judith van Andel, MD, PhD, Francis Tan, MD, Al de Weerd, MD, PhD, Ben Vledder, Wytske Hofstra, MD, PhD, Richard Lazeron, MD, PhD, Ghislaine van Thiel, PhD, Kit C.B. Roes, PhD, Frans Leijten, MD, PhD, and the Dutch Tele-Epilepsy Consortium Neurology ${ }^{\circledR}$ 2018;91:e2010-e2019. doi:10.1212/WNL.0000000000006545

\section{Abstract}

\section{Objective}

To develop and prospectively evaluate a method of epileptic seizure detection combining heart rate and movement.

\section{Methods}

In this multicenter, in-home, prospective, video-controlled cohort study, nocturnal seizures were detected by heart rate (photoplethysmography) or movement (3-D accelerometry) in persons with epilepsy and intellectual disability. Participants with $>1$ monthly major seizure wore a bracelet (Nightwatch) on the upper arm at night for 2 to 3 months. Major seizures were tonic-clonic, generalized tonic $>30$ seconds, hyperkinetic, or others, including clusters $(>30$ minutes) of short myoclonic/tonic seizures. The video of all events (alarms, nurse diaries) and $10 \%$ completely screened nights were reviewed to classify major (needing an alarm), minor (needing no alarm), or no seizure. Reliability was tested by interobserver agreement. We determined device performance, compared it to a bed sensor (Emfit), and evaluated the caregivers' user experience.

\section{Results}

Twenty-eight of 34 admitted participants (1,826 nights, 809 major seizures) completed the study. Interobserver agreement (major/no major seizures) was 0.77 (95\% confidence interval [CI] 0.65-0.89). Median sensitivity per participant amounted to 86\% (95\% CI 77\%-93\%); the false-negative alarm rate was 0.03 per night (95\% CI 0.01-0.05); and the positive predictive value was 49\% (95\% CI 33\%-64\%). The multimodal sensor showed a better sensitivity than the bed sensor ( $\mathrm{n}=14$, median difference 58\%, 95\% CI 39\%-80\%, $p<0.001$ ). The caregivers' questionnaire $(\mathrm{n}=33)$ indicated good sensor acceptance and usability according to 28 and 27 participants, respectively.

\section{Conclusion}

Combining heart rate and movement resulted in reliable detection of a broad range of nocturnal seizures.

\author{
Correspondence \\ Dr. Arends \\ arendsj@kempenhaeghe.nl
}

MORE ONLINE

๑ CME Course

NPub.org/cmelist

From the Academic Center for Epileptology (J.A., C.U., P.C., J.v.D., R.L.); Center for Residential Epilepsy Care (F.T.), Kempenhaeghe, Heeze; Faculty of Electrical Engineering (J.A., C.U., P.C., J.V.D., R.L.), Eindhoven University of Technology; Leiden University Medical Centre (R.D.T.); SEIN-Stichting Epilepsie Instellingen Nederland, Heemstede and Zwolle (R.D.T., T.G., A.d.W., B.V., W.H.); and Brain Center Rudolf Magnus (J.v.A., F.L.), Department of Neurology, and Julius Center for Health Sciences and Primary Care (G.v.T., K.C.B.R.), University Medical Center Utrecht, the Netherlands.

Go to Neurology.org/N for full disclosures. Funding information and disclosures deemed relevant by the authors, if any, are provided at the end of the article. 


\section{Glossary}

ACC $=$ accelerometry; $\mathbf{C I}=$ confidence interval $\mathbf{F N A R}=$ false negative alarm rate $\mathbf{F P A R}=$ false-positive alarm rate $; \mathbf{G T}=$ long generalized tonic; $\mathbf{H K}=$ hyperkinetic; $\mathbf{H R}$ = heart rate; ID = intellectual disability; $\mathbf{O M}=$ other major; $\mathbf{P P V}=$ positive predictive value; SUDEP = sudden unexpected death in epilepsy; TC = tonic-clonic.

About $30 \%$ of people with epilepsy continue to have seizures despite medication and are at high risk for sudden unexpected death in epilepsy (SUDEP). ${ }^{1}$ In particular, nocturnal motor seizures may be dangerous ${ }^{2,3}$ and are often unwitnessed.

Automated seizure detection could improve quality of care, potentially prevent SUDEP, ${ }^{4}$ and provide an objective measurement of nocturnal seizure frequency. ${ }^{5}$

Heart rate (HR) and accelerometry (ACC) are more acceptable for long-term use than EEG. Most detection systems have studied motor activity with ACC or piezoelectric devices during tonic-clonic (TC) seizures in epilepsy monitoring units with sensitivities of $\approx 90 \%$ and false alarm rates ranging from 1 to 10 per 24 hours. ${ }^{6-8}$ Convincing field trials are still lacking ${ }^{9}$ and have so far failed to detect a broader range of seizure types to which caregivers would like to be alerted.

Our consortium performed a number of studies ${ }^{10-13}$ demonstrating the feasibility of combined HR and ACC detection. LivAssured BV (Leiden, the Netherlands) developed a new combined sensor, a bracelet (Nightwatch), worn around the upper arm and measuring HR (by plethysmography) and movement (3-dimensional ACC). We prospectively tested this sensor in a residential setting and compared it to the current standard, a bed sensor. We chose this population because of the high number of TC seizures (hence the increased SUDEP risk); because of the diversity of seizure patterns that can cause complications, including status epilepticus; and because the widely used listening devices are insufficient. ${ }^{14,15}$ Caregivers contributed to the study through the value-sensitive design methodology. ${ }^{16}$

\section{Methods}

\section{Study design}

We conducted a multicenter, prospective, cohort study.

\section{Standard protocol approvals, registrations, and patient consents}

The trial (Dutch Trial Registry 4115) was approved by the Medical Research Ethics Committee of the University Medical Center Utrecht in the Netherlands.

\section{Participants}

The study population consisted of adults with an intellectual disability (ID) who were included if they had a history of $>1$ major nocturnal seizure per month and resided in a long-term facility of 1 of the participating epilepsy centers between
September 1, 2015, and January 9, 2017. Persons with a movement disorder, pacemaker, or skin pigmentation were excluded (the last because we had no proof that the green light--based plethysmography would work on pigmented skin). All participants or their legal guardians provided written informed consent.

\section{Outcome}

\section{Primary}

The primary outcome was performance in terms of sensitivity, positive predictive value (PPV), false-negative alarm rate (FNAR), and false-positive alarm rate (FPAR) of the multimodal sensor to detect major seizures.

\section{Secondary}

The secondary outcomes were a comparison with the bed sensor, the quality of the signal data, factors influencing algorithm performance, and user experience of the caregivers.

\section{Study phases and assessments}

In the first (training) phase of the study, 20 participants were monitored for $\approx 3$ months to test the multimodal sensor (skin contact, skin reactions, ease of use, quality of signals) and to improve the hardware and the detection algorithm. In the second phase, the multimodal sensor generated alarms. Fourteen of these participants entered the second phase. Fourteen new participants (group 2) were added and, after a period of 1 to 2 weeks to become accustomed to the bracelet, were monitored for 2 to 3 months.

The multimodal sensor was compared with the bed sensor in 14 participants who already used the bed sensor before the start of the trial.

\section{Sensor measurements}

The bracelet (figure 1) was fixed around the upper arm on the side where the seizures were known to start. The multimodal sensor settings could not be externally modified. Amplitude and duration of the piezoelectric Emfit bed sensor were optimized according to the manual before the start of the trial. It detects the clonic part of a motor seizure by producing an alarm after a rhythmic movement of 2 to $3 \mathrm{~Hz}$ during 10 to 16 seconds.

\section{Data and events collection}

The HR and ACC signals and the online alarms of the multimodal sensor were wirelessly transmitted to a base station that was connected to a Macbook computer that also was connected to an infrared-sensitive video camera. HR and ACC signals were analyzed offline during phase 1 and online 
Figure 1 Multimodal sensor

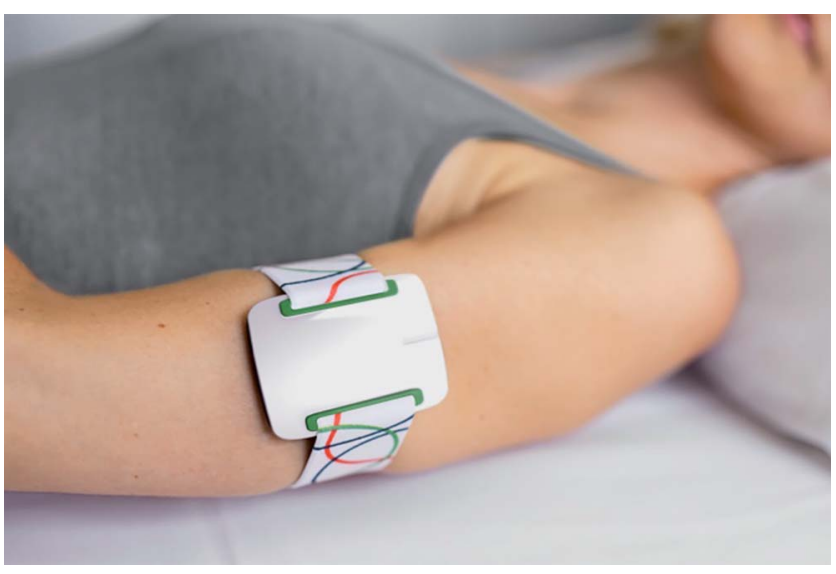

The Nightwatch bracelet contains a photoplethysmographic heart rate module and a 3-dimensional accelerometer. The position on the upper arm was preferred to the wrist because of better signal quality and fewer movement artifacts. The signals or alarms are transmitted by DECT ultralow energy (DECT ULE) directly to the base, which may be connected to a local area network for further transmission of the data and alarms. DECT-ULE is a wireless communication standard with a greater range, reliability, and safety than Bluetooth or Wifi. Figure published with permission from Livassured.

during phase 2, and the video images of the events were annotated with the Nightview program developed specifically for this trial. Sleep and awake times and the nurses' log were entered into the Nightview system. The sleep/wake data were used to set the boundaries for the video review of all logged events and screening nights. A flowchart of the data acquisition process is shown in figure 3 available from Dryad (doi. org/10.5061/dryad.553f4q3). Periods of poor signal quality were excluded by the algorithm. Nights were included only if any data were recorded.

\section{Annotation process and interobserver agreement}

Video images of all reported events (from devices, nurses' records, diaries, and notes) were annotated by the trial nurses. In addition, in a random sample of $10 \%$ of all nights, the complete video recordings were screened to estimate the number of missed major seizures. We considered the following seizure types as clinically urgent and denoted them as major: (1) TC, given the increased SUDEP risk ${ }^{17}$; (2) long generalized tonic ( $\mathrm{GT} ;>30$ seconds), because they may cause respiratory distress; (3) focal hyperkinetic ( $\mathrm{HK})$, given the high risk of injury; and (4) a rest category, other major (OM), consisting of TC-like seizures with atypical semiology and clusters of minor seizures lasting $>30$ minutes. All other seizures were classified as minor and considered false positives. ${ }^{9}$ The trial nurses reviewed the video images in groups of 2 . If they could not agree about the seizure type, they consulted one of the authors (T.G., R.D.T., J.A.) for a final decision.

Video was the gold standard to assess the seizure type. All 6 annotating trial nurses and 2 of the authors (T.G., J.A.) participated in a blinded interobserver agreement evaluation at the end of phase 1 . For this test, 50 random events were selected. The Fleiss $\kappa^{18}$ between major or no major (i.e., minor or no) seizures was the primary outcome measure; secondary outcomes were whether there was a TC seizure and the overall agreement.

\section{Signal processing}

We analyzed the performance, (sensitivity $+\mathrm{PPV}) / 2$, of the algorithm in the preparation phase 1 with the 14 first participants. The algorithm with the best performance was chosen and fixed for use at the start of phase 2 , the prospective trial.

The detection algorithm (see flowchart of the detection algorithm in figure 4 available from Dryad, doi.org/10.5061/ dryad.553f4q3) is executed in the sensor.

In the algorithm, HR values are determined and updated every second on the basis of a 5-minute average of past individual peak-to-peak intervals. Simultaneously, a signal quality index is calculated for each HR value that is based on the plethysmographic waveform. If the signal quality index is adequate $(>80 \%)$, seizure detection starts with a slope and absolute value threshold (tachycardia), depending on the prior baseline HR. If the signal quality index is not adequate, $\mathrm{HR}$ is considered unreliable, and only the ACC algorithm is used for detection. The ACC sensor module provides a motion and a position indicator every second. The motion is a representation of the motor rhythmicity during a seizure and is based on the number of zero crossings for each axis per second. The position parameter indicates whether the patient is lying, sitting, or standing. The function of the position parameter is to prevent alarms when the patient is sitting or standing.

An alarm is generated only if the position indicates lying and the threshold for HR (slope or tachycardia) is exceeded or the motion value stays above threshold for at least 15 seconds.

\section{Performance}

A video event was considered true positive when an alarm was given within 3 minutes before or 5 minutes after the start of a major seizure to allow detection of focal bilateral spreading seizures or atypical seizure types. False-positive detections $<3$ minutes apart were scored as 1. Performance (sensitivity, PPV, FNAR, and FPAR) was calculated per patient and for each sensor (multimodal and bed sensor). FNAR and FPAR were calculated per standardized night (i.e., number of falsenegative or false-positive alarms per 8 hours).

We assessed the relative contributions of HR (slope and tachycardia) and ACC to the true and false detections because many false-positive detections may occur when based on $\mathrm{HR}$ alone. $^{16}$

We assessed the percentage of good signal quality of the HR (>80\%) during all recorded nights. 


\section{Questionnaires}

All caregivers (including those of dropouts) completed a questionnaire designed for this trial that was based on our valuesensitive design study ${ }^{16}$ to evaluate the usability of the multimodal sensor.

\section{Study size and bias}

The sample size of 30 adults was chosen to obtain acceptable confidence limits of the sensitivity and was based on preliminary results of the preceding EEG/video study. ${ }^{8}$ The required sample size for the comparison of the multimodal sensor with the Emfit bed sensor was at least 37 seizures per group, assuming a sensitivity of $80 \%$ for the multimodal sensor and $60 \%$ for the bed sensor $(\beta=0.80, \alpha=0.05)$. Because the participants should have $>1$ seizure per month and the trial lasted 2 to 3 months, we expected $>35$ seizures. Because of the unknown variability of the number of seizures per participant, a more appropriate estimation of the required number of participants was not possible.

Inclusion bias was avoided by using a low inclusion threshold and only a few necessary exclusion criteria.

\section{Data presentation and statistics}

Sensitivity and PPV are used as performance measures mostly at the population level and generally not at an individual level. The strongly varying number of seizures per participant led us to calculate detection rates as averages per person and then use these figures for the descriptive group statistics. Rankorder, nonparametric statistics were used to estimate the median value of parameter values across the average of withinpatient parameters. The reported $95 \%$ confidence intervals (CIs) for the median estimate were calculated from the parameter value that is associated with the closest rank to the rounded $\mathrm{n} \times 0.5 \pm 0.98 \times \operatorname{sqrt}(\mathrm{n})$ rank value. This formula is based on the assumption that the ranks of the patient samples are normally distributed. This assumption is valid for $n$ larger than or on the order of $\mathrm{n}=20$. However, to remain consistent across the presented results, we also used this method for $\mathrm{n}<20$. The 95\% CIs for the interobserver agreement scores were calculated with AgreeStat2015.6 (Advanced Analytics LLC, Gaithersburg, MD). The data are presented as population tables. All individual results, 2 boxplots, and 1 scatterplot are shown in tables 6 through 17 and figures 3 through available from Dryad (doi.org/10.5061/dryad.553f4q3).

The following group comparisons were made: multimodal vs bed sensor and the results of the 10\% screening vs the $90 \%$ nonscreening nights. To test for inclusion bias, group 1 (who already participated in phase 1) was compared to group 2 (those entering the trial in phase 2).

Participants who withdrew from the trial were not replaced.

Statistical tests were performed with the Mann-Whitney test for unpaired data or the Wilcoxon signed-rank test for paired data with $\alpha(2$-sided $)<0.05$.

\section{Data availability}

Individual deidentified participant data are available in the supplemental material. Requests for reanalysis of the complete database, which contains privacy-sensitive information, will have to be approved by the Medical Research Ethics Committee of the University Medical Center Utrecht and the members of the Dutch Tele-Epilepsy Consortium. Data availability is limited by exclusive rights for LivAssured with regard to commercial applications.

\section{Results}

\section{Participants and demography}

During phase 1, 4 withdrawals and 2 exclusions occurred (figure 2 and table 6 available from Dryad, doi.org/10.5061/ dryad.553f4q3).

Demographic and clinical characteristics of the participants entering phase 2 are presented in table 1 . All persons had a generalized or combined generalized/focal type of epilepsy with $>1$ seizure type.

\section{Interobserver agreement}

The agreement between major and other or no seizures was substantial ( $\kappa=0.77,95 \%$ CI $0.65-0.89)$. We also found a substantial agreement between TC and all other major seizure types (GT, HK, OM) $(\kappa=0.62,95 \%$ CI $0.43-0.80)$. The overall agreement between all seizure types (TC, GT, HK, OM, minor, no seizure) was 0.61 (95\% CI 0.51-0.71). All individual scores are shown in table 8 available from Dryad (doi.org/10.5061/dryad.553f4q3).

\section{Performance of the multimodal system}

A graphic representation of the output of the multimodal sensor is shown in figure 5 available from Dryad (doi.org/10. 5061/dryad.553f4q3). The performance of the multimodal sensor is summarized in table 2 .

The total number of observed nights was 1,826 , during which 809 major seizures and 2,040 alarms occurred. Fifty-five of these major seizures (7\%) were difficult to classify. The multimodal sensor had a good median sensitivity per participant (86\%) and a reasonable PPV value (49\%).

One participant had no major seizures all. In 2 others who had 3 major seizures, the seizures were not detected. Three participants had $>1$ false-positive alarm per night $(6.3,3.6$, and 3.2 ), accounting for $75 \%$ of all false alarms (data available from Dryad, table 9, doi.org/10.5061/dryad.553f4q3). Minor seizures accounted for $26 \%$ (368 of 1,402) of the false-positive alarms (data available from Dryad, table 15).

The median FNAR was low (0.03 per night). These falsenegative alarms were calculated over the reported events. Comparison of this result with the data of the completely screened nights (table 2) did not reveal any statistically significant difference. The FPAR showed a high variability. 


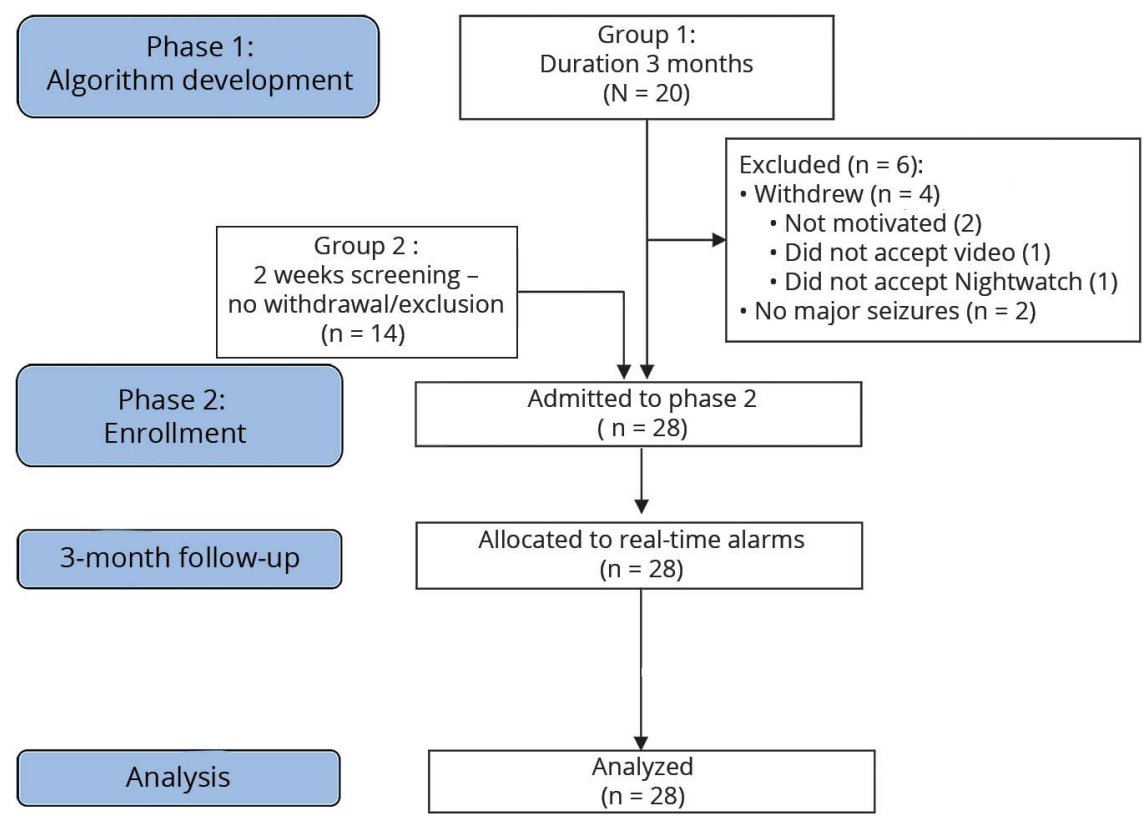

Flowchart according to the Consolidated Standards of Reporting Trials guidelines.

Groups 1 and 2 showed a comparable performance (table 2) despite the fact that the algorithm was fitted to group 1 (median sensitivity $86 \%$ for both groups, PPV $43 \%$ for group 1 and $49 \%$ for group 2 ).

Table 1 Summary of the demographics

\begin{tabular}{lccc}
\hline Demographic data $(\mathbf{n}=\mathbf{2 8})$ & No. & Mean & Range \\
\hline Age, $\mathbf{y}$ & & 29.1 & $15-67$ \\
\hline Sex & & & \\
\hline Female & 10 & & \\
\hline Male & 18 & &
\end{tabular}

IQ level (impairment)

\begin{tabular}{ll}
\hline Mild & 4 \\
\hline Moderate & 11 \\
\hline Severe & 13 \\
\hline
\end{tabular}

Epilepsy type/syndrome

\begin{tabular}{llll}
\hline West/Lennox syndrome & 5 \\
\hline Dravet syndrome & 4 & \\
\hline Other known etiology & 13 & & \\
\hline Unknown etiology & 6 & & $2-5$ \\
\hline Antiepileptic drugs, $\mathbf{n}$ & & 3.3 & \\
\hline Vagus nerve stimulation & 3 & &
\end{tabular}

For individual results, see table 7 available from Dyad (doi.org/10.5061/ dryad.553f4q3).

\section{Comparison with the bed sensor}

In the group who used the multimodal device and the bed sensor $(\mathrm{n}=14)$, the total number of observed nights was 1,097, and 508 major seizures were recorded. The multimodal device generated 877 alarms; the bed sensor, 303. Ten of the 14 patients with both sensors belonged to group 1 with a longer observation period (3 vs 2 months).

The sensitivity of the bed sensor (median 21\%) was significantly lower compared to the multimodal one (median $85 \%$ ) (table 3 ). In 2 participants, no PPV difference could be calculated because no alarms were emitted by either 1 or both sensors. PPV tended to be higher for the bed sensor (median $82 \%$ ) than for the multimodal sensor (median 56\%), but the difference did not reach statistical significance $(p=0.90)$. The bed sensor missed more seizures than the multimodal sensor, with a median FNAR of 0.28 per night vs 0.04 for the multimodal sensor.

\section{Quality of the sensor data}

A good signal quality was obtained in $94 \%$ of total recording time for HR and up to $100 \%$ for ACC. Signal quality could be diminished due to a loss of contact between the bracelet and the skin or by contamination of the plethysmography signal with movement artifacts. No complete nights were excluded because of poor signal quality because a good signal quality of $\mathrm{HR}$ and ACC was required per protocol before the recording was started.

\section{Factors influencing algorithm performance}

\section{HR or ACC detection}

We analyzed how HR and ACC contributed to the performance of the algorithm. The critical modality for the truepositive detections was HR (median detection percentage 
Table 2 Performance of the multimodal sensor

\begin{tabular}{|c|c|c|c|c|c|c|c|c|}
\hline & \multicolumn{2}{|c|}{ Total $(\mathrm{n} \leq 28)$} & \multicolumn{2}{|c|}{ Group $1(n=14)$} & \multicolumn{2}{|c|}{ Group $2(n=14)$} & \multicolumn{2}{|c|}{$10 \%$ Screening $(n \leq 28)$} \\
\hline & Median & $\pm 95 \% \mathrm{Cl}$ & Median & $\pm 95 \% \mathrm{Cl}$ & Median & $\pm 95 \% \mathrm{Cl}$ & Median & $\pm 95 \% \mathrm{Cl}$ \\
\hline Nights, $n$ & 54 & $45-82$ & 90 & $48-98$ & $46^{a}$ & $33-64$ & 6 & $5-9$ \\
\hline Major seizures, n & 14 & $10-26$ & 30 & $13-72$ & $11^{a}$ & $2-14$ & 1.0 & $1-3$ \\
\hline Major seizures per night, $n$ & 0.29 & $0.14-0.41$ & 0.40 & $0.14-0.71$ & 0.22 & $0.02-0.33$ & 0.17 & $0.0-0.38$ \\
\hline Alarms, $n$ & 30.5 & $14-44$ & 53 & 22-109 & $14^{a}$ & $3-35$ & 3 & $1-5$ \\
\hline Sensitivity, \% & 86 & 77-93 & 86 & $64-94$ & 86 & $80-92$ & 100 & $50-100$ \\
\hline PPV, \% & 49 & $33-64$ & 53 & $10-94$ & 49 & $29-56$ & 60 & $0-83$ \\
\hline FNAR per night & 0.03 & $0.01-0.05$ & 0.04 & $0.00-0.21$ & 0.03 & $0.00-0.04$ & 0.00 & $0.00-0.17$ \\
\hline FPAR per night & 0.25 & $0.04-0.35$ & 0.26 & $0.03-0.72$ & 0.18 & $0.02-0.35$ & 0.04 & $0.00-0.33$ \\
\hline
\end{tabular}

Abbreviations: $\mathrm{Cl}$ = confidence interval; FNAR = false-negative alarm rate; FPAR = false-positive alarm rate; PPV = positive predictive value.

Number of recorded nights and seizures and performance of the multimodal sensor are shown. For a boxplot and individual data, see information available from Dyad (doi.org/10.5061/dryad.553f4q3).

a $p<0.05$ (group 1 vs group 2).

92\%, 95\% CI 63\%-96\%), while ACC accounted for only $8 \%$ (95\% CI $0 \%-16 \%)$ of the detections. HR was always the critical modality for the false-positive detections because the ACC modality had no false-positive detections.

\section{Seizure type}

The distribution of the detected major seizure types for the multimodal sensor was $48 \%$ for TC, $21 \%$ for GT, $24 \%$ for $\mathrm{HK}$, and $7 \%$ for OM. TC seizures were well detected by the multimodal sensor (median detection rate 0.96 ). The other seizure types were somewhat less well detected (median detection rates between 0.73 and 0.89 ) (table 4).

\section{Bed sensor}

The bed sensor detected a minority of all major seizure types (medians between 0.00 for $\mathrm{OM}$ and 0.30 for HK seizures), including the TC type (0.14). We did not expect this because the bed sensor records the length of rhythmic movements and assumes TC seizure to be present if the length of this rhythmic movement exceeds a programmable value between 10 and 16 seconds.

\section{Outcomes of questionnaires}

An assessment questionnaire was designed that addressed the key aspects of the multimodal sensor that were identified through a process of value-sensitive design. ${ }^{14}$ Thirty-three respondents (30 nurses, 2 parents, and 1 not specified), for the 28 participants in phase 2 and for 5 of the 6 excluded participants, completed the questionnaire (table 5).

Caregivers indicated that the sensor implementation facilitated a more timely response to urgent situations, while the burden of

Table 3 Comparison of the multimodal and the bed sensor

\begin{tabular}{|c|c|c|c|c|c|c|}
\hline & \multicolumn{2}{|c|}{ Multimodal sensor } & \multicolumn{2}{|c|}{ Bed sensor } & \multicolumn{2}{|c|}{ Multimodal - bed sensor } \\
\hline & Median & $\pm 95 \% \mathrm{Cl}$ & Median & $\pm 95 \% \mathrm{Cl}$ & Median & $\pm 95 \% \mathrm{Cl}$ \\
\hline Nights, $n$ & 79 & 48-99 & 79 & 48-99 & & \\
\hline Major seizures, $\mathbf{n}$ & 21 & $12-72$ & 21 & $12-72$ & & \\
\hline Major seizures per night, $n$ & 0.34 & $0.12-0.71$ & 0.34 & $0.12-0.71$ & & \\
\hline Alarms, $n$ & 46 & $13-108$ & 22 & $1-30$ & & \\
\hline Sensitivity, \% & 85 & $71-93$ & 21 & $6-32$ & $58^{a}$ & $39-80$ \\
\hline PPV, \% & 56 & $34-94$ & 82 & $30-90$ & 2 & $(52)-7$ \\
\hline FNAR per night & 0.04 & $0.00-0.17$ & 0.28 & $0.08-0.54$ & $(0.21)^{a}$ & $(0.49)-(0.11)$ \\
\hline FPAR per night & 0.23 & $0.00-0.59$ & 0.03 & $0.00-0.21$ & 0.11 & $(0.11)-0.45$ \\
\hline
\end{tabular}

Abbreviations: $\mathrm{Cl}$ = confidence interval; FNAR = false-negative alarm rate; FPAR = false-positive alarm rate; $P P V=$ positive predictive value. For a boxplot, a scatterplot of the differences, and individual data, see information available from Dyad (doi.org/10.5061/dryad.553f4q3).

a $p<0.05$ (multimodal - bed sensor). 
Table 4 Detection rates of multimodal and bed sensor for each seizure type

\begin{tabular}{|c|c|c|c|c|}
\hline Detection rate per seizure type & TC & GT & HK & MO \\
\hline \multicolumn{5}{|l|}{ Multimodal sensor } \\
\hline No. & 22 & 11 & 5 & 14 \\
\hline Median & 0.96 & 0.89 & 0.73 & 0.84 \\
\hline$-95 \% \mathrm{Cl}$ & 0.80 & 0.33 & 0.50 & 0.50 \\
\hline$+95 \% \mathrm{Cl}$ & 1.00 & 1.00 & 1.00 & 1.00 \\
\hline \multicolumn{5}{|l|}{ Bed sensor } \\
\hline No. & 12 & 7 & 3 & 5 \\
\hline Median & 0.14 & 0.17 & 0.30 & 0.00 \\
\hline$-95 \% \mathrm{Cl}$ & 0.06 & 0.00 & 0.27 & 0.00 \\
\hline$+95 \% \mathrm{Cl}$ & 0.31 & 0.38 & 0.32 & 0.43 \\
\hline
\end{tabular}

Abbreviations: $\mathrm{Cl}=$ confidence interval; $\mathrm{GT}=$ long generalized tonic (>30 seconds); $\mathrm{HK}=$ hyperkinetic; $\mathrm{OM}=$ other major; $\mathrm{TC}=$ tonic-clonic.

care remained unchanged. A considerable number of sensor problems was reported. All of these were transient or have been resolved. Skin irritation occurred during the preparatory phase of the trial and was eliminated by removing the paint on the surface of the sensor and by avoiding the bracelet being fixed too tightly. Nonfitting of the bracelet was prevented by changing the texture and attachment of the elastic band. Nonacceptance by the participant occurred twice; it was temporary in one and led to exclusion in another. The majority of caregivers $(n=20)$ experienced more freedom, but only a minority (10) thought the participants had gained more autonomy.
In addition, respondents gave the user-friendliness of the multimodal sensor a grade of 7.3 on a scale from 1 to 10 (1 being the lowest user-friendliness). With regard to privacy, respondents graded the level of invasion of privacy as 4.2 on the same scale ( 1 being the lowest level of invasion).

\section{Discussion}

This long-term prospective study demonstrates that it is possible to reliably detect major motor seizures using a combination of HR and ACC parameters. The multimodal sensor

Table 5 Assessment of key valued aspects of the multimodal sensor

\begin{tabular}{|c|c|c|c|c|c|}
\hline \multicolumn{6}{|c|}{ Questionnaire for parents/caregivers } \\
\hline Aspect & Item & Yes, $n$ & No, $n$ & Other, $\mathrm{n}$ & Not applicable/no answer, $n$ \\
\hline \multirow[t]{4}{*}{ Care } & Helps caregiver provide better care & 22 & 5 & 5 & 1 \\
\hline & More timely care & 18 & & & \\
\hline & Increased understanding of daytime behaviors & 4 & & & \\
\hline & Helps relieve burden of caregiving & 7 & 26 & - & - \\
\hline \multirow[t]{5}{*}{ Sensor } & Sensor is accepted by the patient & 28 & 2 & 2 & 1 \\
\hline & Problems with the sensor & 13 & 8 & & 12 \\
\hline & Skin irritation & 3 & & & \\
\hline & Armband does not fit & 5 & & & \\
\hline & Poor connection or signal reception & 5 & & & \\
\hline \multirow[t]{3}{*}{ Usability } & Sufficient technical support & 27 & 1 & 5 & - \\
\hline & Sufficient with installation, not for use & 2 & & & \\
\hline & Sufficient for use, not with installation & 3 & & & \\
\hline \multirow[t]{2}{*}{ Autonomy } & Offers more autonomy to people with epilepsy & 10 & 13 & - & 10 \\
\hline & Offers caregiver more freedom & 20 & 11 & - & 2 \\
\hline
\end{tabular}


has a much better sensitivity than the bed sensor and a comparable PPV. It is well accepted, is easy to use, and provides accurate measurements over time. The device can also be used to monitor epilepsy treatment.

Conventional studies have been testing rhythmic movements in epilepsy monitoring units where the majority of patients are screened for epilepsy surgery. These patients usually have focal seizures of temporal lobe origin that evolve into classic TC seizures. The bed sensor ${ }^{6}$ and other systems based on movement detection ${ }^{7}$ perform well under these circumstances, but their performance deteriorates in the specific population of this trial. This relatively low performance of the bed sensor is in accordance with the results of the ACC part of our algorithm. Accelerometric research ${ }^{11}$ and clinical experience indicate that people with ID and epilepsy show more atypical seizure patterns: different sequences of tonic and clonic phases may occur in changing order, and status epilepticus may develop from seizure types other than classic TC. This may explain the low performance of the bed sensor in this population, although the sensor had been selected before the trial as the best alternative.

Despite the low performance, bed sensors and other rhythmic movement-based sensors remain useful: they may yield accurate alarms if rhythmic movements last for 10 to 16 seconds or longer and the sensitivity is adapted to avoid false positives. ${ }^{6}$ The low performance of the bed sensor confirms the need to include signal modalities other than ACC in seizure detection. Physiologically, complementary signal combinations are as follows: HR or skin conductance (both expressions of autonomic function), EMG (for tonic phases of seizures), ${ }^{7,10,19}$ and video ${ }^{20}$ or audio detection (based on specific sounds). ${ }^{14}$ We chose HR, the fastest signal other than $\mathrm{EEG}^{2,21}$ that is considered an adequate alternative to EEG. ${ }^{22}$ Measurements of HR in smartwatches are less reliable because of the oval form of the wrist, which may prevent optimal body contact. Under controlled conditions, smartwatches record useful signals up to $88 \%$ of the time, ${ }^{23}$ while our device showed good signal quality for $94 \%$, i.e., half the error rate ( $6 \%$ vs $12 \%)$. Results of the rhythmic movement-based seizure detection at the wrist by smartwatches are disappointing and in line with our results for the bed sensor. ${ }^{24,25}$ Contrary to smartwatches, our device does not need a smartphone interface. Its DECT technology provides a larger wireless range $(50 \mathrm{~m})$ than Bluetooth $(10 \mathrm{~m})$. Respiratory function (depressed postictal blood oxygen saturation) is also used for seizure detection with fairly good results, ${ }^{26}$ but the obtrusiveness and false-positive responses make it less useful for long-term detection at home. The plethysmographic signal also has the potential to detect breathing rate. ${ }^{27}$

We introduced the concept of major seizures as a representation of clinical urgency of seizures. ${ }^{10}$ Major seizures are specified by medical professionals as seizure semiology, which includes loss of consciousness, falls, long duration, intense movements, respiratory problems, cluster or status, salivation/vomiting, oxygen drop, migraine, or visual problems. ${ }^{28}$ All 3 seizure types (TC, GT, HK) and the less well-defined class OM are characterized by
$>1$ of these elements and were adequately detected by our device as a result of the introduction of the HR signal. The ACC module of the device detected a minority of the seizures compared to the HR module. This is due to the high threshold imposed by the requirement of 15-second continuous rhythmic movement, causing seizures with a short clonic phase to be missed, but also a PPV of $0 \%$. A shorter duration will increase the detection rate of the ACC module but also will introduce false alarms. Regarding the PPV of the multimodal device, we have to consider different factors than for the bed sensor. The aspecific nature of the HR response and the atypical evolution of motor seizures in people with epilepsy and $\mathrm{ID}^{11}$ may lower the PPV for the multimodal device, while the required long duration of the clonic part of the seizures ${ }^{6}$ may result in a higher PPV for the bed sensor. Together, these may explain why a slightly higher PPV was found for the bed sensor, although this was not statistically significant. The PPV results for group 1 , in whom the algorithm was developed, and group 2 were similar, suggesting that our results are not liable to overfitting.

Although generic " 1 size fits all" solutions are feasible, ${ }^{10}$ personalized algorithms need to be explored in future. ACC and HR seem complementary: contrary to rhythmic movements, the HR increase is not linked to 1 particular seizure expression but is the result of the sympathetic response to seizures. We did not systematically study the interactions between the HR and ACC signals. HR increases may cause false-positive detections in persons with high resting HRs or strong excitability such as children. ${ }^{29}$ Our HR-related algorithm might benefit from self-learning algorithms accounting for physiologic arousals ${ }^{30}$ and varying baseline levels and requires further study. Adding other signal modalities to the 2 used in this study may also help to improve our algorithms. ${ }^{19}$

The decision to consider minor seizures as false-positive results was made to avoid too many alarms for the caregiver and to limit the alarms to those requiring immediate care. While these minor seizures could be valuable to improve the reliability of the seizure counts, we currently have no algorithm that can discriminate these events from nonepileptic arousals or movements during sleep. In our study population, focal seizures other than HK occurred relatively rarely. ${ }^{11}$ Thus, we cannot predict whether the HR and motor patterns in these seizures will be detected. Novel algorithms might benefit from analysis of HR variability to discriminate between epileptic and nonepileptic seizures ${ }^{31}$ or to use HR variability as a marker of cardiac arousability. ${ }^{32}$ Daytime use of detectors when the higher level of activity may cause more false positives is also an important issue.

The multimodal sensor might be of help in preventing SUDEP. A recent guideline of the American Association of Neurology ${ }^{33}$ indicates that clinicians may use a remote listening device to reduce SUDEP risk in high-risk individuals (Level C). ${ }^{34}$ Although comparative studies are lacking, combining HR and ACC seems more effective for detecting major 
seizures because of the shortcomings of current audio detection. $^{14,15}$

Seizure diaries are an inaccurate, subjective method for counting seizures. ${ }^{35}$ Our device may offer a more objective method for nocturnal seizure counting not only to use in clinical trials but also to measure the efficacy of individual therapy. The multimodal sensor may provide data about the urgency of seizures because we found indications that the height and duration of the HR increase correlate with the subjectively estimated urgency of clinical seizures. ${ }^{36}$

Our study has a number of limitations. First, we did not use video-EEG as the gold standard. The decision to use video alone was imposed by the long duration of the monitoring $(\geq 3$ months) and the residential use outside the hospital. The substantial interobserver agreement confirms that this decision was justified. Second, the exclusion of persons with abnormal movements prevents extrapolation of the results of this study to this group, consisting mainly of persons with perinatal damage leading to choreatiform movement patterns. Third, because of the current intensity of the light-emitting source, we could not reliably measure the light reflections in people with dark-colored skin. This can be overcome by adaptive light intensity. Fourth, the assessment of quality of life is important for measuring the economic gain. In this population, however, the standard tools for quality of life are not adequate: the low intellectual level (moderate to severe impairment) prevents adequate communication and is often associated with immobility and other handicaps. The results of the value-based scale (gain in care, freedom of movement for the caregiver, and presumed autonomy for the user) were counterbalanced by the reported mild interference with the person's privacy. These results, however, cannot be compared with other studies because of the new methodology. ${ }^{16}$ Fifth, interference with privacy needs to be explored further because video verification may be used more often in the relatively small number of users with many (false) alarms.

\section{Author contributions}

Statistical analysis conducted by P.C., J.A., and K.C.B.R. (epidemiologist, University Medical Center Utrecht).Other contributions: J.A., R.D.T, TG, C.U., J.V.D., J.v.A., A.d.W., B.V., G.v.T., K.C.B.R., F.T., and F.L. contributed to the study design. J.v.A. and J.A. did the literature search. Data were obtained by T.G., B.V., C.U., P.C., J.A., R.D.T., W.H., R.L., G.v.T., and P.C. P.C. and J.A. created the figures. All authors contributed to the interpretation of results, reviewed and critically revised the article, and approved the final version for submission.

\section{Study funding}

This study was funded by the following grants: A Disease Management Chronic Diseases grant from the Dutch National Science Foundation (NWO-ZonMW) No. 300040003, the NUTS-Ohra Foundation No. 1203-050, and the Dutch Epilepsy Foundation for extra material costs. LivAssured, the company developing the Nightwatch device, has obtained an exclusive license to implement or use the data in the future for commercial purposes or in commercial enterprises in exchange for a percentage of the revenue for the institutes. None of the authors has shares in the aforementioned company nor in the aforementioned institutes, nor has any of them received nor will any of them receive any compensation referring to future sales of the Nightwatch. The Dutch Tele-Epilepsy Consortium will receive more research funds from the institutes as a consequence of this license.

\section{Disclosure}

The authors report no disclosures relevant to the manuscript. Go to Neurology.org/N for full disclosures.

\section{Publication history}

Received by Neurology January 2, 2018. Accepted in final form August 9, 2018.

\section{References}

1. Löscher W, Schmidt D. Modern antiepileptic drug development has failed to deliver: ways out of the current dilemma. Epilepsia 2011;52:657678

2. Lamberts RJ, Thijs RD, Laffan A, Langan Y, Sander JW. Sudden unexpected death in epilepsy: people with nocturnal seizures may be at highest risk. Epilepsia 2012;53 253-257.

3. Van der Lende M, Hesdorffer D, Sander JW, Thijs RD. Nocturnal supervision and SUDEP risk at different epilepsy care settings. Neurology Epub 21 Sept 2018.

4. Ryvlin P, Nashef L, Lhatoo SD, et al. Incidence and Mechanisms of Cardiorespiratory Arrests in Epilepsy Monitoring Units (MORTEMUS): a retrospective study. Lancet Neurol 2013;12:966-977.

5. Hoppe C, Poepel A, Elger CE. Accuracy of patient seizure counts. Arch Neurol 2007; 64:1595-1599.

6. Van Poppel K, Fulton SP, McGregor A, Ellis M, Patters A, Wheless J. Prospective study of the Emfit movement monitor. J Child Neurol 2013;28:1434-1436.

7. Ulate-Campos A, Coughlin F, Gaínza-Lein M, et al. Automated seizure detection systems and their effectiveness for each type of seizure. Seizure 2016;40:88-101.

8. Van Andel J, Thijs RD, De Weerd A, Arends J, Leijten F. Non-EEG based ambulatory seizure detection designed for home use: what is available and how will it influence epilepsy care? Epilepsy Behav 2016;57:82-89.

9. Beniczky S, Ryvlin P. Standards for testing and clinical validation of seizure detection devices. Epilepsia 2018;59:9-13.

10. Van Andel JM, Ungureanu C, Arends JB, et al. Multimodal, automated detection of nocturnal motor seizures at home: is a reliable seizure detector feasible. Epilepsia Open 2017;2:424-431.

11. Nijsen TM, Arends JB, Griep PA, Cluitmans PJ. The potential value of threedimensional accelerometry for detection of motor seizures in severe epilepsy. Epilepsy Behav 2005;7:74-84.

12. Van Elmpt W, Nijsen T, Griep P, et al. A model of heart rate changes to detect seizures in severe epilepsy. Seizure 2006;15:366-375.

13. Massé F, Penders J, Serteyn A, Van Bussel MJ, Arends JB. A Miniaturized Wireless ECG-Monitor for Real-Time Detection of Epileptic Seizures. New York: Wireless Health; 2010:111-117.

14. Arends JB, van Dorp J, van Hoek D, et al. Diagnostic accuracy of audio-based seizure detection in people with epilepsy with severe epilepsy and an intellectual disability. Epilepsy Behav 2016;62:180-185.

15. Van der Lende M, Cox F, Visser G, Sander JW, Thijs RD. Value of video monitoring for nocturnal seizure detection in a residential setting. Epilepsia 2016;57:1748-1753.

16. Van Andel J, Leijten F, van Delden H, van Thiel G. What makes a good home-based nocturnal seizure detector? A value sensitive design. PLoS One 2015;10:e121446.

17. Devinsky O, Hesdorffer DC, Thurman DJ, Lhatoo S, Richerson G. Sudden unexpected death in epilepsy: epidemiology, mechanisms, and prevention. Lancet Neurol 2016;15:1075-1088

18. Landis JR, Koch GG. The measurement of observer agreement for categorical data. Biometrics 1979;33:159-174.

19. Beniczky S, Conradsen I, Henning O, Fabricius M, Wolf P. Automated real-time detection of tonic-clonic seizures using a wearable EMG device. Neurology 2018;90: e428-e434.

20. Geertsema E, Gutter T, Vledder B, et al. Automated video-based detection of convulsive seizures in a residential care setting. Epilepsia 2018;59:53-60.

21. Hirsch M, Altenmüller DM, Schulze-Bonhage A. Latencies from intracranial seizure onset to ictal tachycardia: a comparison to surface EEG patterns and other clinical signs. Epilepsia 2015;56:1639-1647.

22. Osorio I, Manly BF. Is seizure detection based on EKG clinically relevant? Clin Neurophysiol 2014;125:1946-1951.

23. Jovanov E. Preliminary analysis of the use of smartwatches for longitudinal health monitoring. Conf Proc IEEE Eng Med Biol Soc 2015:865-868. 
24. Reeder B, David A. Health at hand: a systematic review of smart watch uses for health and wellness. J Biomed Inform 2016;63:269-276.

25. Patterson AL, Mudigoudar B, Fulton S, et al. SmartWatch by SmartMonitor: assessment of seizure detection efficacy for various seizure types in children: a large prospective single-center study. Pediatr Neurol 2015;53:309-311.

26. Goldenholz DM, Kuhn A, Austermuehle A, et al. Long-term monitoring of cardiorespiratory patterns in drug-resistant epilepsy. Epilepsia 2017;58:77-84.

27. Cernat RA, Ciorecan SI, Ungureanu C, Arends J, Strungaru R, Ungureanu GM. Recording system and data fusion algorithm for enhancing the estimation of the respiratory rate from photoplethysmogram. Conf Proc IEEE Eng Med Biol Soc 2015; 2015:5977-5980.

28. Van de Vel A, Smets K, Wouters K, Ceulemans B. Automated non-EEG based seizure detection: do users have a say? Epilepsy Behav 2016;62:121-128.

29. Fleming S, Thompson M, Stevens R, et al. Normal ranges of heart rate and respiratory rate in children from birth to 18 years of age: a systematic review of observational studies. Lancet 2011;377:1011-1018.

30. De Cooman T, Varon C, Van de Vel A, et al. Adaptive nocturnal seizure detection using heart rate and low-complexity novelty detection. Seizure 2018;59:48-53.
31. Ponnusamy A, Marques JLB, Reuber M. Comparison of heart rate variability parameters during complex partial seizures and psychogenic nonepileptic seizures. Epilepsia 2014;53:1314-1321.

32. Liu H, Yang Z, Huang L, Qu W, Hao H, Li L. Heart-rate variability indices as predictors of the response to vagus nerve stimulation in patients with drug-resistant epilepsy. Epilepsia 2017;58:1015-1022.

33. Harden C, Tomson T, Gloss D, et al. Practice guideline summary: sudden unexpected death in epilepsy incidence rates and risk factors: report of the Guideline Development, Dissemination, and Implementation Subcommittee of the American Academy of Neurology and the American Epilepsy Society. Neurology 2017;88:1674-1680.

34. Langan Y, Nashef L, Sander JW. Case-control study of SUDEP. Neurology 2005;64: $1131-1133$.

35. Blachut B, Hoppe C, Surges R, Elger C, Helmstaedter C. Subjective seizure counts by epilepsy clinical drug trial participants are not reliable. Epilepsy Behav 2017;67:122-127.

36. Van Andel J, Gutter T, Ungureanu C, Arends J, Leijten F. A comparison of heart rate patterns in clinically urgent and non-urgent nocturnal motor seizures. In: Van Andel J, editor. Towards a Multimodal System for Nocturnal Seizure Detection. PhD thesis. Utrecht: University of Utrecht; 2015. 


\section{Neurology}

\section{Multimodal nocturnal seizure detection in a residential care setting: A long-term prospective trial}

Johan Arends, Roland D. Thijs, Thea Gutter, et al.

Neurology 2018;91;e2010-e2019 Published Online before print October 24, 2018

DOI 10.1212/WNL.0000000000006545

\section{This information is current as of October 24, 2018}

\section{Updated Information \&} Services

References

Citations

Subspecialty Collections

Permissions \& Licensing

\section{Reprints}

including high resolution figures, can be found at: http://n.neurology.org/content/91/21/e2010.full

This article cites 32 articles, 3 of which you can access for free at: http://n.neurology.org/content/91/21/e2010.full\#ref-list-1

This article has been cited by 1 HighWire-hosted articles: http://n.neurology.org/content/91/21/e2010.full\#\#otherarticles

This article, along with others on similar topics, appears in the following collection(s):

Clinical trials Observational study (Cohort, Case control)

http://n.neurology.org/cgi/collection/clinical_trials_observational_stud y_cohort_case_control

Epilepsy monitoring

http://n.neurology.org/cgi/collection/epilepsy_monitoring_

Information about reproducing this article in parts (figures,tables) or in its entirety can be found online at:

http://www.neurology.org/about/about_the_journal\#permissions

Information about ordering reprints can be found online:

http://n.neurology.org/subscribers/advertise

Neurology ${ }^{\circledR}$ is the official journal of the American Academy of Neurology. Published continuously since 1951, it is now a weekly with 48 issues per year. Copyright Copyright @ 2018 The Author(s). Published by Wolters Kluwer Health, Inc. on behalf of the American Academy of Neurology.. All rights reserved. Print ISSN: 0028-3878. Online ISSN: 1526-632X.

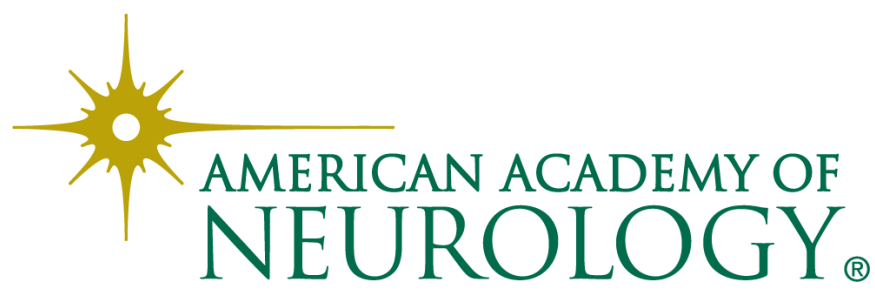

